\title{
Topology optimisation of micro fluidic mixers considering fluid-structure interactions with a coupled Lattice Boltzmann algorithm
}

\author{
David J. Munk ${ }^{\mathrm{a}, *}$, Timoleon Kipouros ${ }^{\mathrm{b}}$, Gareth A. Vio ${ }^{\mathrm{a}}$, Grant P. Steven ${ }^{\mathrm{a}}$, \\ Geoffrey T. Parks ${ }^{\mathrm{b}}$ \\ ${ }^{a}$ The University of Sydney, Sydney, 2006, Australia \\ ${ }^{b}$ University of Cambridge, Cambridge, CB2 1PZ, United Kingdom
}

\begin{abstract}
Recently, the study of micro fluidic devices has gained much interest in various fields from biology to engineering. In the constant development cycle, the need to optimise the topology of the interior of these devices, where there are two or more optimality criteria, is always present. In this work, twin physical situations, whereby optimal fluid mixing in the form of vorticity maximisation is accompanied by the requirement that the casing in which the mixing takes place has the best structural performance in terms of the greatest specific stiffness, are considered. In the steady state of mixing this also means that the stresses in the casing are as uniform as possible, thus giving a desired operating life with minimum weight.

The ultimate aim of this research is to couple two key disciplines, fluids and structures, into a topology optimisation framework, which shows fast convergence for multidisciplinary optimisation problems. This is achieved by developing a bi-directional evolutionary structural optimisation algorithm that is directly coupled to the Lattice Boltzmann method, used for simulating the flow in the micro fluidic device, for the objectives of minimum compliance and maximum vorticity. The needs for the exploration of larger design spaces and to produce innovative designs make meta-heuristic algorithms, such as genetic
\end{abstract}

\footnotetext{
${ }^{*}$ Corresponding author

Email address: david.munk@sydney.edu.au (David J. Munk)
}

Preprint submitted to Journal of Computational Physics

August 4, 2017 
algorithms, particle swarms and Tabu Searches, less efficient for this task.

The multidisciplinary topology optimisation framework presented in this article is shown to increase the stiffness of the structure from the datum case and produce physically acceptable designs. Furthermore, the topology optimisation method outperforms a Tabu Search algorithm in designing the baffle to maximise the mixing of the two fluids.

Keywords: structural topology optimisation, design-dependent loads, fluid-structure interactions, pressure loading problems, LBM, multidisciplinary analysis

\section{Nomenclature}

$\begin{array}{ll}\text { 2D } & \text { Two-dimensional } \\ 3 \text { D } & \text { Three-dimensional } \\ \text { BESO } & \text { Bi-directional Evolutionary Structural Optimisation } \\ \text { BGK } & \text { Bhatnagar-Gross-Krook } \\ \text { CPU(s) } & \text { Computational Processing Unit (Seconds) } \\ \text { DNS } & \text { Direct Numerical Simulation } \\ \text { ESO } & \text { Evolutionary Structural Optimisation } \\ \text { FEA } & \text { Finite Element Analysis } \\ \text { FEM } & \text { Finite Element Method } \\ \text { FSI } & \text { Fluid-Structure Interactions } \\ \text { LBE } & \text { Lattice Boltzmann Equation } \\ \text { LBM } & \text { Lattice Boltzmann Method } \\ \text { SED } & \text { Strain Energy Distributions } \\ \text { SIMP } & \text { Solid Isotropic Material with Penalisation } \\ \text { TS } & \text { Tabu Search }\end{array}$

\section{Introduction}

Over the last decade topology optimisation of continuum structures [1] has 5 matured to a level where it is slowly becoming a common design tool used by 
industry [2]. The main idea is to find the optimal distribution of the material in a predefined design domain considering an objective function and constraints. Since its introduction [3], a wide variety of objective functions have been applied with topology optimisation algorithms, diversifying their application to almost all fields of engineering and design [4, 5, 6]. However, many applications are still open to research, such as design-dependent pressure loading problems [7].

Traditional topology optimisation methods seek to find the maximum stiffness with a predefined fixed loading $[8,9,10]$. However, there are many applications in which the load location and magnitude vary as the design changes during the optimisation process. One such application can be found in the design of micro fluidic mixers. Micro fluidic devices have two major fields of application: firstly, in mechanical systems as flow control systems, pressure pumps and many more; and secondly, as medical diagnostic devices, where the expression lab on a chip is commonly used [11. Many medical technologies, such as cell biology and protein crystallization, have advanced because of micro fluidics. Therefore, optimisation of such devices is imperative to maintain and improve their impact across various fields. The implementation of topology optimisation to the design of micro fluidic devices is limited due to the many design iterations required, which necessitates the reduction of the problem size in order 25 to achieve acceptable execution times [12]. The problem is further complicated by Fluid-Structure Interactions (FSI) present between the mixing device and the flow, making the design dependent on the pressure loading from the fluid. The challenge in optimising a structure with an applied pressure load lies in determining the loading surface on which the pressure acts. This becomes more difficult for traditional density-based topology optimisation methods, such as Solid Isotropic Material with Penalisation (SIMP) [13] and homogenization [3]. In these methods, the structural boundaries, and hence loaded surfaces, are not explicitly defined due to the presence of intermediate density elements [14].

In this article, an extended Bi-directional Evolutionary Structural Optimi35 sation (BESO) algorithm is applied to the design of micro fluidic mixers considering FSI. A three-dimensional (3D) Lattice Boltzmann method (LBM) is 
used as the flow solver with two fluid species, extending beyond the basic twodimensional (2D) Stokes flow used in the literature. This presents a practical example not yet seen in structural optimisation of design-dependent pressure-

40 loaded problems with fluid-structure interfaces. Finally, a novel formulation for the objective of viscosity maximisation is presented. To the best of the authors' knowledge, topology optimisation for viscosity maximisation is applied here for the first time with an evolutionary topology optimisation procedure. With the proposed framework, the design of micro fluidic devices can be easily implemented with high fidelity algorithms incorporated at the conceptual and detailed design phases, efficiently coupling multiple disciplines.

The rest of this article is organised as follows. Sect. 2 outlines the necessary background and literature for the manuscript. Sect. 3 presents the governing equations for the fluid and structural models. Sect. 4 presents the topology 50 optimisation problems and outlines the BESO method employed in this work. In Sect. 5, the methodology for coupling the multiple disciplines and extending the optimisation methods to the fluid-structure problem is outlined, along with the introduction of the novel sensitivity analysis for vorticity maximisation. The test case studied is then given in Sect. 6 along with a validation of the Lattice Boltzmann method. The results from the BESO algorithm are given in Sect. 7 for the structural optimisation problem with design-dependent loads and for the viscosity maximisation problem using a novel sensitivity analysis. Finally, Sect. 8 concludes the article.

\section{Background}

A significant effort in attempts to solve topology optimisation problems considering design-dependent pressure loads has been in the creation of the loading surface using continuous topology optimisation techniques. In the literature, several methods exist to achieve this; however, they can primarily be arranged into two groups. The first group seeks to identify a fluid-structure boundary and directly apply the loads onto the finite elements, such as is seen in 
[14, 15, 16, 17. Conversely, the second group of methods models the pressure loading with alternative physics or utilise mixed formulations to avoid explicitly defining a loading surface. Such examples can be seen in the work of Chen and Kikuchi [18, which uses a fictitious thermal loading and a dryness coefficient to identify the fluid and solid regions, or that of Zheng et al. [19, where they introduced a potential function modelled on the electric potential and applied a fictitious electric field. Thus, the literature shows that the classic element density-based topology optimisation algorithms become onerous when dealing with FSI coupled systems.

An alternative branch of topology optimisation, which lends itself to the application of design-dependent pressure loads, is based on discrete methods. Yang et al. 20] applied evolutionary methods [21] to the design of structures, which included structural downward surface loads. They extend the BESO method to applications in fluid-loaded structural problems. Recently, Picelli et al. [7] extended this method to the application of general movable fluid-structure interfaces with design-dependent pressure loads. Later, Picelli et al. 22] applied this method to topology optimisation problems for frequency maximisation considering acoustic-structure interactions. The discrete update scheme present in evolutionary methods allows the use of separate modules for the fluid and structural domains with different governing equations. This overcomes a well-studied challenge associated with the classic density-based methods, dealing with moving multiphysics loads and interfaces. Therefore, discrete methods such as BESO present great potential applications in the areas of multiphysics optimisation.

Level-set methods have also been applied to solve pressure loading problems [23, 24, 25, 26], having the advantage that material boundaries are implicitly defined. Challis and Guest [27] propose a level-set method for the optimisation of fluid flow. They show that the discrete nature of the optimisation problem leads to significant advantages over density-based topology optimisation algorithms. Furthermore, the no-slip boundary condition can be implemented directly, which is accurate and removes the need for interpolation schemes and continuation methods. This gives notable computational savings, since it only 
requires flow to be modelled in fluid regions.

From this literature it is evident that, although methods for design-dependent pressure loads have been studied, most examples given are two-dimensional, by the following equation:

$$
\mathbf{f}=[\mathbf{K}] \mathbf{u}
$$

where $\mathbf{f}$ and $\mathbf{u}$ are the nodal force and displacement vectors respectively, and $[\mathbf{K}]$ is the global stiffness matrix of the structure. The element stiffness matrices 
125

only the Dirichlet boundary conditions are applied to the solid domain, such that:

$$
\mathbf{u}=\mathbf{u}_{0} \quad \text { on } S_{0}
$$

where $\mathbf{u}_{0}$ is the vector of prescribed displacements along the boundary of the structure defined by $S_{0}$. Therefore, Eq. 1 can be solved along with the boundary conditions (Eq.2) to get the structural displacements, $\mathbf{u}$, as a result of an applied load, $\mathbf{f}$.

For further discussion of the finite element method, the interested reader is advised to seek out the work of Chandrupatla and Belegundu [32].

\subsection{Fluid model}

Compared to structural dynamics, fluid dynamics has only recently been considered in topology optimisation. Since its inception, in evolutionary [5] and continuous 33 . algorithms, several methods have been considered for modelling the fluid domain. Typically, a fluid problem is discretised by finite volume, finite element or discontinuous Galerkin methods [34. These methods have difficulty with complex 3D geometries, since they require fine-body-fitted meshes. Moreover, these approaches are not easily applied to multiphase flows with variable complex interfaces. Thus, the literature of structural topology optimisation with fluid pressure loads is limited to simple Stokes flow models and 2D cases [7.

In contrast, Lattice Boltzmann methods are built around the concept of 145 solving a discrete Boltzmann equation on Cartesian grids. Therefore, complex geometries can be easily handled with the LBM [35]. Further, the LBM has been proven to converge to the incompressible Navier-Stokes equations for the low Mach number regime, and recently has gained broad recognition for the simulation of micro fluids, multi-phase problems and flows through porous media 35, 36, 37, 38]. The LBM has, thus far, seen limited application in topology optimisation of flows [27, 39, 40, 41, 42], and, furthermore, it has not yet been coupled with structural topology optimisation with design-dependent fluid pressure loads. 
Motivated by the previous success of the optimisation of flow channels [27.

155

160 , a 3 D LBM method is used as the fluid model in this work. The code has been validated against Navier-Stokes simulations using commercial codes [44] and experimental analysis [45], thus proving the stability and accuracy of this method. The fundamental concept of the LBM is to construct kinetic models, based on Newton's laws, which incorporate the essential physics of microscopic processes, such that the macroscopic processes are correctly modelled. Hence, the fluid is considered as a finite number of molecules, the motion of which is governed by Newton's laws of dynamics. Since the number of molecules of a particular gas in a volume of one metre cubed is usually in the order of Avogadro's number, it is near impossible to simulate all particles individually [29, 46]. Therefore, the averages over a sample of molecules are simulated, passing from a microscopic to a mesoscopic scale, making it possible to obtain macroscopic values, such as fluid density and velocity, from the moments of the velocity distribution functions that express the distribution state of the particles.

The LBM solves a discretised Boltzmann equation, known as the Lattice Boltzmann Equation (LBE), which can represent macroscopic properties by incorporating velocity distribution functions. The lattice used in this work is the D3Q19 lattice, meaning three dimensions and 18 moving particles per node with one rest node. The Boltzmann equation can be represented using the velocity distribution function, $f(x, t, \gamma)$, by:

$$
S h \frac{\partial f}{\partial t}+\gamma \cdot \nabla f=Q(f)
$$

175 gas particle position and velocity respectively, and $Q$, known as the collision operator, exhibits the effect of collisions between the particles. For simplicity and without losing any generality, the Bhatnagar-Gross-Krook (BGK) collision model [4] is employed in this work; where the BGK collision model is formulated

$$
Q(f)=-\frac{1}{\tau_{B}}\left(f-f^{e q}\right)
$$

here $\tau_{B}$ is the dimensionless relaxation time, which represents the average time 
until the next collision. $f^{e q}$ is a Maxwell distribution and can be expressed as a local equilibrium to the Boltzmann equation:

$$
f^{e q}=\frac{\rho}{T^{\frac{d}{2}}} \exp \left(-\frac{|\gamma-u|^{2}}{T}\right)
$$

where $\rho$ and $u$ represent the fluid density and velocity respectively, $T$ is the temperature, assumed constant to satisfy the isothermal condition, and $d$ is the number of spatial dimensions. From kinetic theory, it follows that the macroscopic variables in the flow field can be derived from the moments of the velocity distribution function with respect to the velocity field, $E$, such that:

$$
\rho=\int_{E} f d \gamma, \quad u=\frac{1}{\rho} \int_{E} \gamma f d \gamma
$$

The fundamental concept of the LBM is the discretisation of the infinite set of particle velocities, $\gamma$, in Eq. 3. This allows the calculation of macroscopic quantities from the moments of a finite number of velocity distribution functions to be obtained as solutions to the Navier-Stokes equations. Thus, the discretisation of the Boltzmann equation (Eq. 3) in time and space at the lattice site $x$ is found by:

$f_{\alpha}\left(x+\gamma_{\alpha} \cdot \Delta t, t+\Delta t\right)-f_{\alpha}(x, t)=-\frac{1}{\tau_{B}} \cdot\left[f_{\alpha}(x, t)-f_{\alpha}^{e q}(x, t)\right] \quad$ for $\alpha=0, \ldots, 18$

where $\Delta t$ is the time step and $\alpha$ is the number of particles per node. If the velocity is low or the flow has a low Mach number, the discrete local equilibrium distribution function, $f_{\alpha}^{e q}$, obtained by the Maxwell distribution (Eq. 5), can be approximated as the Taylor expansion [48]:

$$
f_{\alpha}^{e q}=w_{\alpha} \rho\left[1+3 \cdot \gamma_{\alpha} \cdot u+\frac{9}{2}\left(\gamma_{\alpha} \cdot u\right)^{2}-\frac{3}{2} \gamma_{\alpha} \cdot \gamma_{\alpha}\right]
$$

where $w_{\alpha}$ are the weights as reported in [49]. The density, $\rho$, and fluid velocity, $u$, are obtained from the following moments of the velocity distribution functions:

$$
\rho=\sum_{\alpha=0}^{18} f_{\alpha}, \quad u=\frac{1}{\rho} \sum_{\alpha=0}^{18} \gamma_{\alpha} f_{\alpha}
$$


The left-hand side of the discretised Boltzmann equation performs the streaming operation: the particles move from one node to the nearest neighbours along the velocity directions, $\alpha$. The right-hand side represents the collision term, evaluated using the BGK collision model (Eq. 4), and describes the redistribution of the particles at each node at every time step. The LBE is solved according to these two processes, where the collision step is evaluated as:

$$
f_{\alpha}^{\text {new }}=f_{\alpha}(x, t)-\frac{1}{\tau_{B}} \cdot\left[f_{\alpha}(x, t)-f_{\alpha}^{e q}(x, t)\right]
$$

where $f_{\alpha}^{e q}(x, t)$ is evaluated using the Taylor expansion of the Maxwell distribution function (Eq. 8). The second step is the streaming operation, which means the transfer of the particle distribution, $f_{\alpha}^{n e w}(x, t)$, to the particle distribution function at the next time step:

$$
f_{\alpha}^{n e w}\left(x+\gamma_{\alpha} \cdot \Delta t, t+\Delta t\right)=f_{\alpha}^{n e w}(x, t)
$$

The relaxation time, $\tau_{B}$, is related to the kinematic viscosity, $\kappa$, of the fluid via:

$$
\kappa=\frac{2 \cdot \tau_{B}-1}{6}
$$

Therefore, the relaxation time is limited to $\tau_{B}>0.5$, since this constrains the viscosity to be always positive. Consequently, the following LBE is obtained:

$$
f_{\alpha}^{n e w}\left(x+\gamma_{\alpha} \cdot \Delta t, t+\Delta t\right)=f_{\alpha}(x, t)-\frac{1}{\tau_{B}} \cdot\left[f_{\alpha}(x, t)-f_{\alpha}^{e q}(x, t)\right]
$$

Eq. 13 is solved to simulate the fluid dynamics of the system. The total number of iterations used for the LBM simulations is 4000, since stability has been demonstrated and validated against Navier-Stokes simulations using a commercial code, ANSYS ${ }^{\circledR}$ CFX [44], and experimental analysis [45].

For a further discussion of the Lattice Boltzmann method, the interested reader is advised to seek out the textbook by Succi 29 .

\section{Topology optimisation}

The first part of this work is concerned with compliance minimisation of micro fluidic mixers under fluid pressure loading with volume constraints. The 
objective is to find the distribution of a given amount of material to obtain a structure with maximum stiffness. Therefore, the topology optimisation problem for this case can be stated as:

$$
\begin{array}{cl}
\text { Minimise: } & \frac{1}{2} \mathbf{u}^{T}[\mathbf{K}] \mathbf{u} \\
\text { subject to: } & {[\mathbf{K}] \mathbf{u}=\mathbf{f}} \\
& \sum_{i=1}^{n} x_{i} \leq V \\
& \mathbf{x}=[0,1]
\end{array}
$$

where $\mathbf{x}$ is the vector of design variables, $x_{i}, n$ is the total number of elements in the model and $V$ is a predefined volume fraction. Since a discrete algorithm is used, the design variable, $x_{i}$, is forced to become discrete where $x_{i}=1$ represents solid and $x_{i}=0$ represents void or fluid material.

The second part of this work is concerned with vorticity maximisation of micro fluidic mixers for a given Reynolds number and volume fraction. The objective is to find the topology of the mixer that gives the highest vorticity in the mixing region. Therefore, the topology optimisation problem for this case is given by:

$$
\begin{array}{ll}
\text { Minimise: } & -\vec{\omega} \\
\text { subject to: } & R e=R e_{0} \\
& \sum_{i=1}^{n} x_{i} \leq V \\
& \mathbf{x}=[0,1]
\end{array}
$$

where $\vec{\omega}$ is the vorticity of the flow in the mixing region, Re is the Reynolds number of the flow, and $R e_{0}$ represents a predefined Reynolds number. For this case, a design variable of $x_{i}=1$ represents fluid elements, whereas $x_{i}=0$ represents solid elements.

\subsection{Evolutionary Structural Optimisation}

The original Evolutionary Structural Optimisation (ESO) algorithm is monotonic, meaning elements can only be removed from the design domain [21]. 

terial from the initial, over-sized, structure, gradually evolving towards the optimal design [50. Although the ESO method has been applied to a wide range of problems [51, 5], it is limited by only removing material from the structure. The consequences of this are that the initial model must be significantly overSubsequent ESO methods, referred to as BESO, allow material, if deemed beneficial, to be re-admitted to the design domain [52. Modern BESO algorithms are convergent and mesh-independent [53, simultaneously removing and adding material from and to the design domain until the constraints and a convergence

\subsection{Mesh independency and checkerboarding}

In FEA, the change in the stiffness of the structure due to the removal of an element is equal to the element strain energy [55]. This change is defined as the element sensitivity for the compliance minimisation problem:

$$
\alpha_{e}=\frac{\partial c}{\partial x_{i}}=\frac{1}{2} p x_{i}^{p-1} \mathbf{u}_{e}^{T}[\mathbf{K}]_{e} \mathbf{u}_{e}
$$

where $c$ is the compliance, $p=3$ is the penalisation factor, and the subscript $e$ 270

represents elemental values. To smooth the element sensitivity numbers across the entire domain, a filter scheme is used that alleviates the problem of meshindependency and checker-boarding, which result from the sensitivity numbers 
becoming discontinuous across the element boundaries. The filter scheme is similar to that presented by Sigmund and Petersson [56]; however, nodal sensitivity numbers are used when calculating the updated element sensitivity numbers based on the surrounding structure. The nodal sensitivity numbers are defined as the average of the element sensitivity numbers connected to the node:

$$
\alpha_{n_{j}}=\sum_{i=1}^{M} w_{i} \alpha_{e_{i}}
$$

where $M$ is the number of elements connected to the $j^{\text {th }}$ node and $\alpha_{e_{i}}$ is the $i^{\text {th }}$ element sensitivity number (Eq. 16). The weighting factor of the $i^{t h}$ element, $w_{i}$, is defined with respect to its distance from the $j^{t h}$ node, as:

$$
w_{i}=\frac{1}{M-1}\left(1-\frac{r_{i j}}{\sum_{i=1}^{M} r_{i j}}\right)
$$

where $r_{i j}$ is the distance from the centre of the $i^{t h}$ element to the $j^{\text {th }}$ node. The nodal sensitivity numbers (Eq. 17) are then converted to smooth element sensitivities using a mesh-independency filter. A filter radius, $r_{m i n}$, is defined to identify the nodes that will have an effect on the element sensitivity. The value of $r_{\min }$ must be large enough such that the sub-domain, $\Omega$, covers at least one element. For the purpose of this study, $r_{\min }=3$ times the spacing of the LBM lattice and must remain constant for all mesh densities. Nodes located inside $\Omega$ contribute to the smoothing of the element sensitivity, by:

$$
\alpha_{e_{i}}=\frac{\sum_{j=1}^{N} w\left(r_{i j}\right) \alpha_{n_{j}}}{\sum_{j=1}^{N} w\left(r_{i j}\right)}
$$

where $N$ is the total number of nodes in the sub-domain, $\Omega$, and $w\left(r_{i j}\right)$ is the linear weighting factor, defined as:

$$
w\left(r_{i j}\right)=r_{\text {min }}-r_{i j} \quad j=1,2, \ldots, N
$$

The filter scheme smooths the nodal sensitivity numbers over the entire design domain, including void regions. Therefore, it effectively addresses the mesh-dependency and checkerboard problems. However, the objective function and corresponding topology may not be convergent. In order to overcome this 
295

where $i t r$ is the current iteration number. Therefore, the updated sensitivity number includes the history of the sensitivity information from previous iterations.

\subsection{Convergence}

The BESO method defines a target volume for each iteration, defined as:

$$
V_{i t r+1}=V_{i t r}(1 \pm E R)
$$

where $E R$, known as the evolutionary ratio, is a percentage of the current structural volume, and increases or decreases $V_{i t r+1}$ towards the desired volume constraint, $V$, defined in Eq. 14. This, in turn, sets the threshold, $\alpha_{t h}$, of the sensitivity numbers. Therefore, solid elements are switched to void/fluid when:

$$
\alpha_{e_{i}} \leq \alpha_{t h}
$$

and void/fluid elements are switched to solid when:

$$
\alpha_{e_{i}}>\alpha_{t h}
$$

The amount by which the volume of the structure can increase between iterations, $A R$, is restricted by a maximum addition ratio $A R_{\max }$. Once $A R>$ $A R_{\max }$, only the elements with the highest sensitivity numbers are added, such that $A R=A R_{\max }$. Then the elements with the lowest sensitivity numbers are removed, in order to satisfy the target volume $V_{i t r+1}$. Void/fluid elements can have a higher sensitivity than solid elements due to the soft material model, which adds a small fictitious stiffness to these elements in the structural analysis. Due to the mesh-dependency filter, void/fluid elements near solid regions with high sensitivity numbers have their sensitivities increased by the elements inside their sub-domain $\Omega$. 
Once the volume constraint is satisfied, the iteration target volume remains constant at $V$. The topology evolves until a convergence criterion is satisfied. being applied to the topology. The current topology and loads are passed onto the Finite Element Method (FEM) module, which defines the structural boundary conditions and outputs the displacements and compliance of the structure. 
This is passed onto the topology optimisation algorithm, which calculates the sensitivities and updates the topology of the structure for the next iteration.

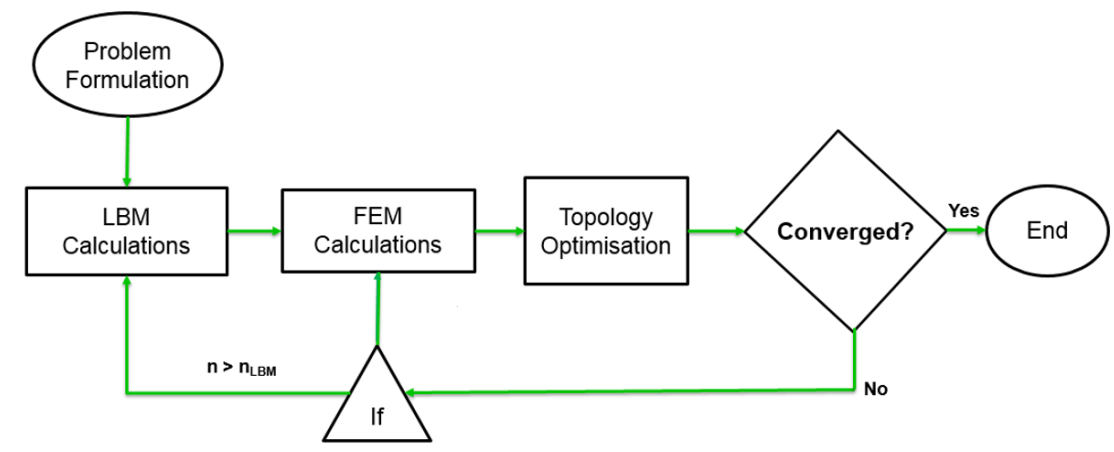

Fig. 1. Numerical framework for coupled LBM-topology optimisation.

The numerical framework (Fig. 1) provides two possible ways of looping. The first is between the topology optimisation algorithm and the FEM module, which is performed at every iteration in the optimisation process. The second loop passes the updated topology back through the LBM flow solver. The main computational burden of the numerical framework (Fig. 1) is the LBM solver. Therefore, the computational efficiency can be increased by restricting the second loop to only be performed every $n_{L B M}$ iterations. However, this reduces the degree of coupling, and hence, the impact of the FSI on the algorithm. Thus, it

355 is left for future work to quantify the impact of this coupling on the objective and computational efficiency of the algorithm. Further, to reduce the computational burden of the coupled LBM-topology optimisation method, identical grids are used in the LBM and FEA analyses, thereby avoiding complex, timeinefficient, spline methods to transfer the loads between the structural and fluid modules and re-meshing of the domains [58].

\subsection{Compliance sensitivity analysis}

For the design of micro fluidic devices, modifying an element leads to changes in the load vector, which need to be considered in the sensitivity analysis. The 
sensitivity analysis (Eq. 16) is therefore modified, such that the change in the

load vector is considered [20, 7]:

$$
\alpha_{e}=\frac{1}{2} p x_{i}^{p-1} \mathbf{u}_{e}^{T}[\mathbf{K}]_{e} \mathbf{u}_{e}+p x_{i}^{p-1} \mathbf{u}_{e}^{T} \Delta \mathbf{f}_{e}
$$

where $\Delta \mathbf{f}_{e}$ is the change in the element load vector. Taking the isoparametric bilinear elements used in this work, the load change in one element for a fluid pressure load is given by:

$$
\Delta \mathbf{f}_{e}=\frac{1}{4} p_{i} A_{i}\{1,0,0,0,0,0,1,0,0,0,0,0 \ldots)_{24 \times 1}^{T}
$$

where $p_{i}$ is the pressure load on the element and $A_{i}$ is the elemental area. It is assumed that the flow travels from left to right, i.e. in the positive $x$ direction, and that the baffle plate is perpendicular to the flow, as shown in Fig.2, Eq. 26] is similar to the sensitivity analysis derived in [7, with some noticeable differences, however. First, the algorithm in [7] is two-dimensional, so the flow and solid domains are parallel, not perpendicular, as is the case here. This means that the components of the load vector change. In contrast, in this study the magnitude of the load and the surface it acts on change. However, the direction of the load remains constant, as it is always perpendicular to the surface, since the problem is three-dimensional [20, 59, and, in consequence, a coupling matrix is not required [7].

Another difference between the sensitivity analysis of this work (Eq. 26) and [7] is that Picelli et al. use a hard-kill BESO algorithm, whereas a soft-kill algorithm is used here. Thus, the sensitivity analysis derived in [7] is discrete, i.e. it only has two states, whereas in this work the design variable, $x_{i}$, must be included. The sensitivity analysis has therefore been modified for 3D and continuous topology optimisation problems.

\subsection{Vorticity sensitivity analysis}

The second problem analysed in this work is maximising the vorticity, or mixing of the two fluid species, for a given Reynolds number and volume fraction (Eq. 15). This objective is imperative to the operation of micro fluidic mixers 

have low Reynolds numbers, typically lower than 1000, vorticity is an efficient measure of the degree of mixing, as shown in the work of 60] or [45]. Recently, Romero and Silva 61] used the method of moving asymptotes to optimise the shape of the channel between two blades of a rotor to minimise the energy dissipation and vorticity. They noted that the combination of advanced numerical techniques, such as computational fluid dynamics and topology optimisation, can be an efficient tool to obtain a significant enhancement in the rotor design 61. Kasumba and Kunisch 62 propose the use of a translation invariant cost functional for the reduction of vortices in the context of shape optimisation for a fluid flow domain. They derive different shape sensitivity derivatives based on different cost functionals, showing striking differences in the optimal shapes found using the different derivations 62. In this work, the vorticity is measured using the circulation method, which computes a circulation integral over the eight nearest neighbour velocities. This method was chosen over the leastBy using this objective function (Eq. 28), vortices in the flow can be thought of as regions of high vorticity magnitude. Therefore, the sensitivity numbers are the reciprocal vorticity values evaluated at each element, since we want to maximise (rather than minimise) the vorticity, which gives:

$$
\alpha_{e}=\max (\vec{\omega})-\Delta \gamma_{e}^{T} x_{i}^{p-1} \Delta \gamma_{e}
$$

415

where $\Delta \gamma_{e}$ is the change of the element velocity vector, defined as:

$$
\gamma_{e}=\left\{\Delta \gamma_{x}, \Delta \gamma_{y}, \Delta \gamma_{z}, W_{x}, W_{y}, W_{z}\right\}^{T}
$$


where $x, y$ and $z$ are the spatial components and $W$ is the circulation. Therefore, fluid elements are present where the vorticity values are high and solid elements are formed in regions of low vorticity, such that the constraint on the predefined volume fraction, $V$, is satisfied. The sensitivity number (Eq. 30 is then used in the BESO formulation, outlined in Sect. 4, to obtain the topology of the micro fluidic mixer that gives a maximum vorticity.

\section{Models and validation}

This section presents the results of the BESO algorithm (Sect. 4) for the structural optimisation problem with design-dependent loads. First, the case study analysed in this work is detailed. This is followed by a validation of the LBM solution with previous numerical and experimental studies [4, 45]. Finally, the coupled results are given along with an analysis of the improvement in the objective versus the computational expense of the algorithm.

\subsection{Case study}

The model used in this study is a baffled micro-reactor, as depicted in Fig. 2 The model consists of a tubular vessel fitted with a fuel inlet tube, located coaxially in the main vessel, and a multi-holed baffle plate through which the oxidiser is introduced. The fluid domain and layout of the micro-reactor model are shown in Fig. 2

The dimensions of the fluid domain (Fig. 22) are expressed in LBM nodes, where the dimensions of the lattice are $680 \times 73 \times 73$ lattice units $(2795 \times 300 \times 300$ $\mu \mathrm{m})$, with additional nodes used for the wall, in the $x, y$ and $z$ directions, respectively. The baffle is located 60 lattice units $(300 \mu \mathrm{m})$ downstream of the flow inlet (Fig. 2). The imposed inlet conditions are the velocities of the flow in the inlet tube and annulus area. At the outlet a convective boundary condition is applied, based on the velocity. The no-slip condition at the walls is implemented by modelling them as full-way bounce-back. The mass flow rate between the inner tube and annulus is set to $5 \%$ to mimic the experiments performed by Moghtaderi [45]. 

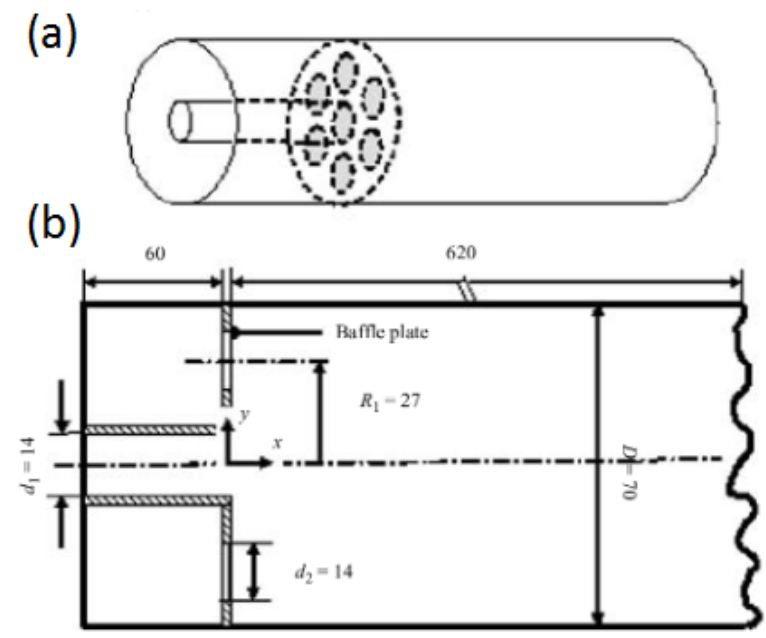

(c)

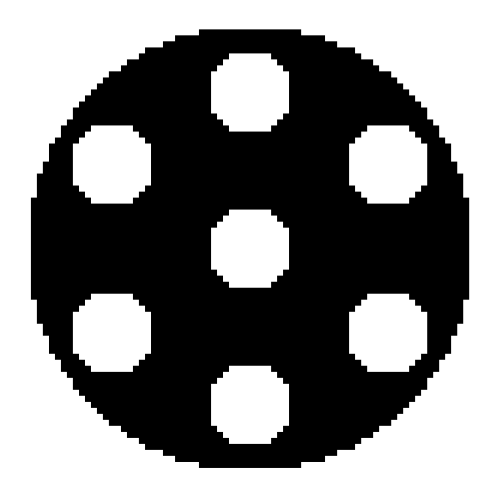

Fig. 2. (a) Fluid domain 64]; (b) Layout of micro-reactor model 64]; (c) Multi-holed baffle plate.

In this section, the topology optimisation algorithm (Sect. 4) is applied to the multi-holed baffle plate (Fig. 22) to maximise its stiffness for a given volume fraction. FEA (Sect. 3.1) is therefore performed on the baffle only. The plate is modelled using four-node quadrilateral elements, with all six degrees of freedom active, so membrane, bending and transverse shear stresses are present. A clamped boundary condition, i.e. all six degrees of freedom are restrained, is applied along the boundary of the baffle. The boundary of the central hole is designated as non-designable for the topology optimisation, since this is de- 
termined by the fuel line and inlet conditions, which have been constrained in the fluid domain (Fig. 2a) to be identical to the previous numerical [44] and experimental [45] studies.

\subsection{Validation of Lattice Boltzmann method}

To validate the present LBM simulations, the test case (Fig. 2) with the multi-holed baffle plate is performed for various baffle $\left(d_{2}\right)$ and fuel inlet $\left(d_{1}\right)$ diameters. The same test case was used in previous numerical [60, 44] as well as experimental [45] studies. Therefore, this provides a baseline for the present study. Fig. 3 shows the velocity map from this study for the reference geometry as defined in [45] $\left(d_{1}=d_{2}=60 \mu \mathrm{m}\right.$ and $R_{1}=75 \mu \mathrm{m}$, see Fig. 22).

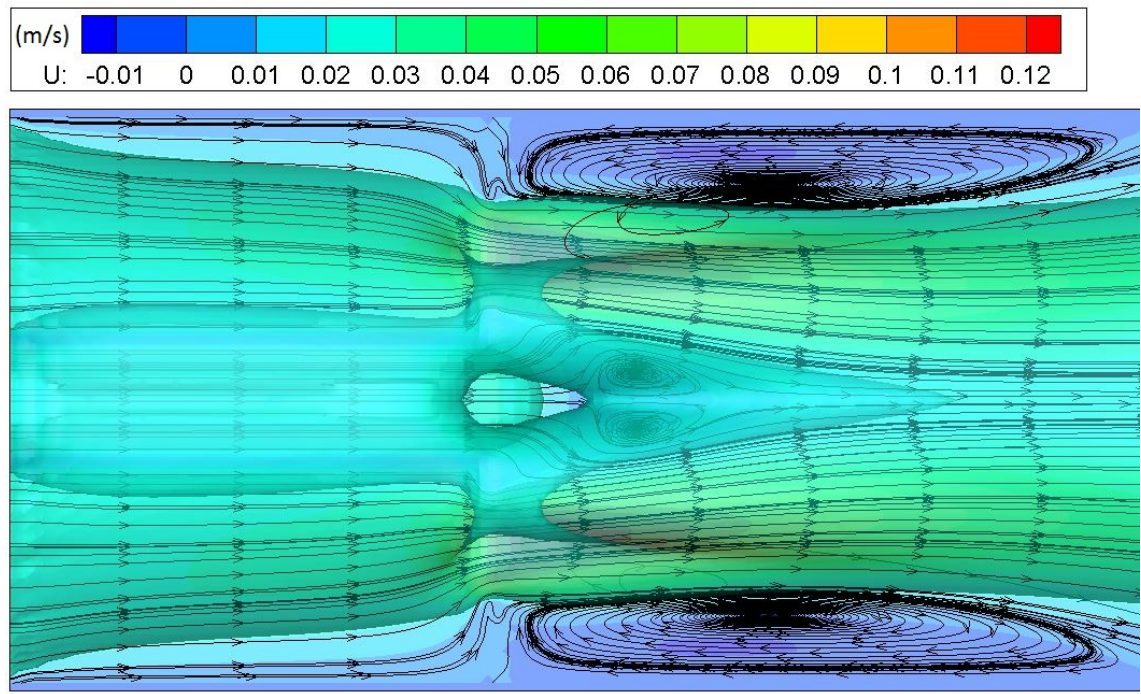

Fig. 3. Velocity map for micro fluidic mixer with the reference geometry.

The velocity vectors show two pairs of counter-rotating regions of recirculation, one pair immediately after the baffle, one region is at the top and the other at the bottom of the mixer, and another pair just after the exit of the fuel inlet line (Fig. 3). This flow pattern is similar to that demonstrated by the experiments in [45], where the recirculation zones and reverse flow near the central tube outlet was observed. Hence, this agreement indicates that the LBM 
simulations are able to reproduce the flow characteristics of the micro fluidic mixer.

Both the experimental study by Moghtaderi et al. 45, and the numerical study by Woodfield et al. 60] examined the impact of the baffle plate geometry on the mixing phenomena. First, the influence of the fuel inlet diameter $\left(d_{1}\right)$ on the reverse flow region immediately after the exit of the fuel inlet line is investigated. The velocity map from this study with a fuel inlet diameter reduced by $50 \%\left(d_{1}=30 \mu \mathrm{m}\right)$ from that of the reference geometry is illustrated in Fig. 4 .
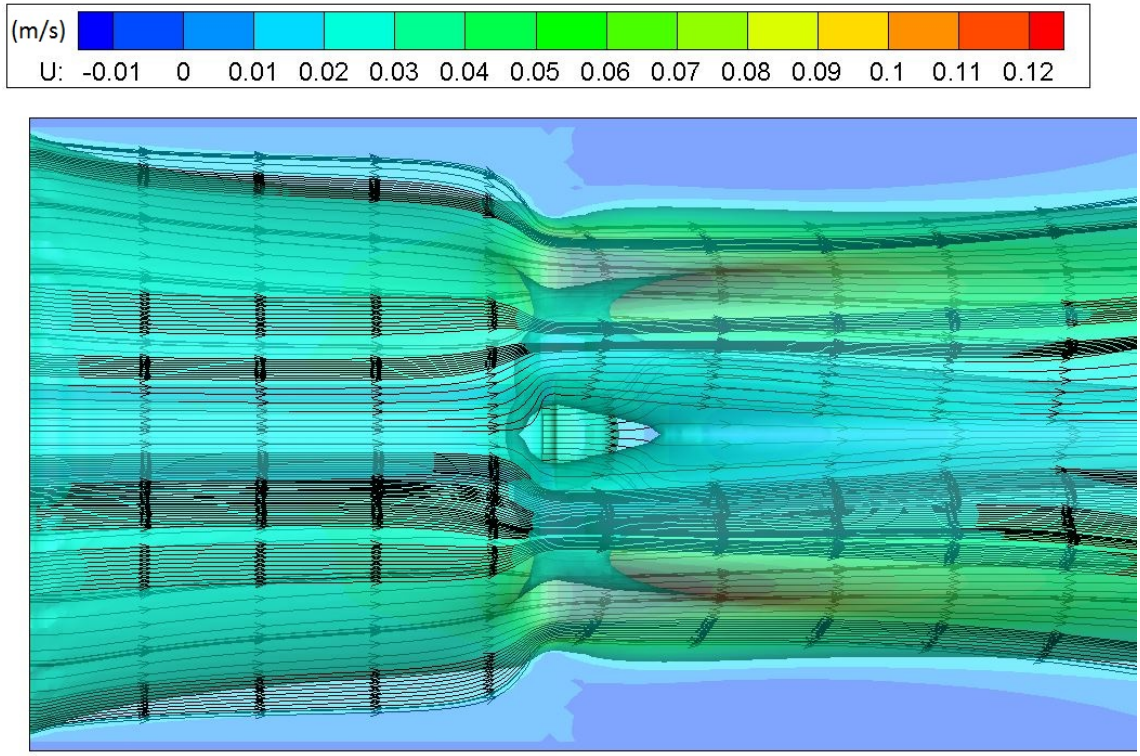

Fig. 4. Velocity map for micro fluidic mixer with a $50 \%$ reduction in the fuel inlet diameter.

The results from this study show that the effect of the reduced fuel inlet diameter on the reversed flow is to reduce the recirculation and reverse flow zones after the exit of the fuel inlet line (Fig. 4). Therefore, the amount of mixing compared with the reference geometry (Fig. 3) is reduced. This behavior was also observed in the experimental results of [45. They attributed this to the reduction in the fuel inlet diameter increasing the velocity of the flow in the fuel line. These results are also in agreement with the numerical simulations of 60]. They too showed that the reverse flow and recirculation region is reduced 
when the fuel inlet tube diameter is reduced. Lastly, the impact of the baffle hole diameters $\left(d_{2}\right)$ on the flow characteristics of the micro fluidic mixer is studied. The velocity map from this study with a $50 \%$ reduction in the baffle hole diameters is given in Fig. 5

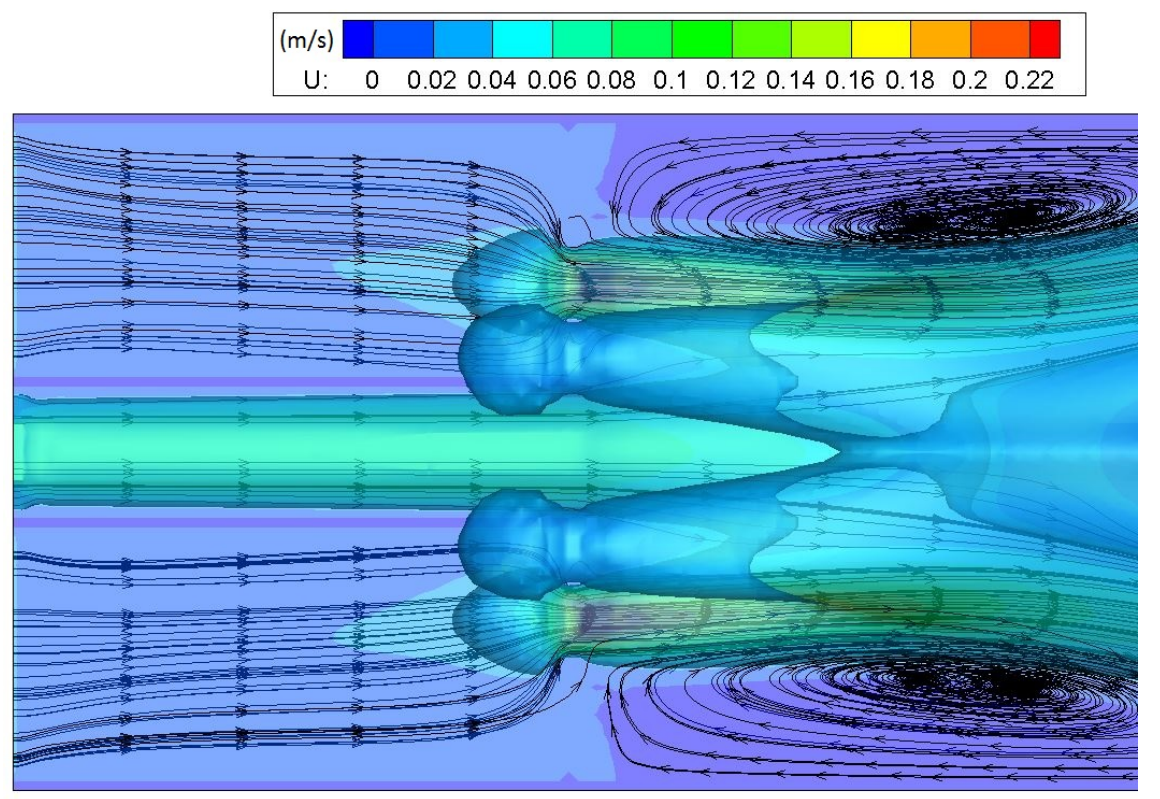

Fig. 5. Velocity map for micro fluidic mixer with a $50 \%$ reduction in the baffle hole diameter.

The velocity through the baffle holes is greatly increased when the diameters are reduced (Fig. 5). The effect of this is to cause a reduction in the reverse flow and recirculation zone at the outlet of the fuel line compared with the reference geometry (Fig. 3). However, the other pair of counter-rotating recirculation zones, also seen in the reference geometry case, have, for this case, become larger. A similar behaviour was found in the experimental results of [45]. They showed that any deviation from the reference geometry results in a significant departure from the flow characteristics of the initial case. Likewise, in the numerical study of [60], reducing the baffle hole sizes produced an increase in the velocities and an expansion in the reverse flow region, eliminating the recirculation zone at the exit of the fuel line. Therefore, it is suggested that, for improved mixing, 

the material around the baffle is distributed more evenly, reducing the overal strain energy density in the structure. The strain energy distributions for the initial and final topologies are given in Fig. 7 


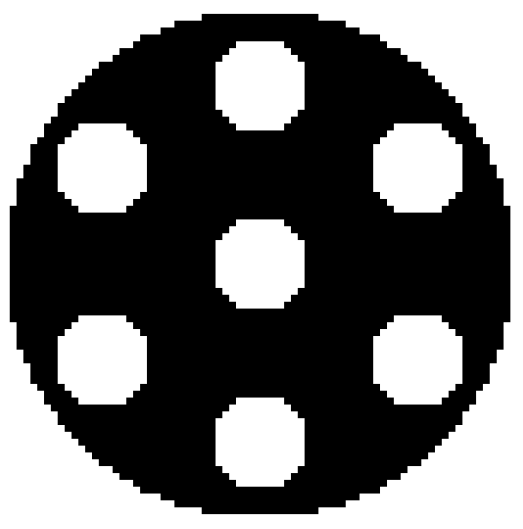

(a) Initial

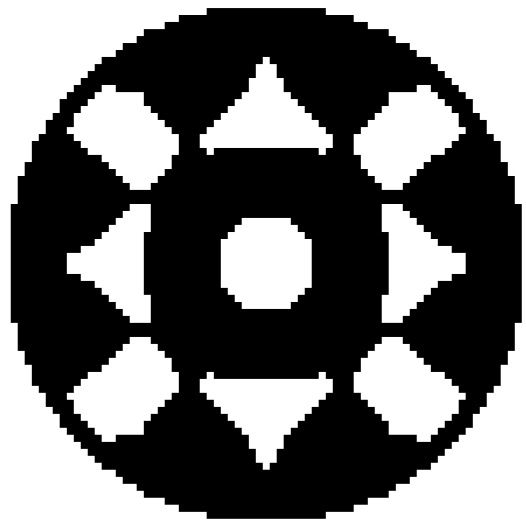

(b) Final

Fig. 6. Initial and final BESO-found topologies for the baffle with design-dependent loading for compliance minimisation test case.

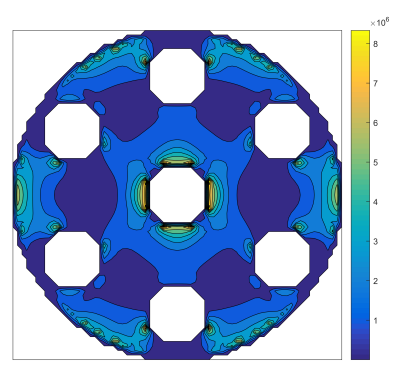

(a) Initial

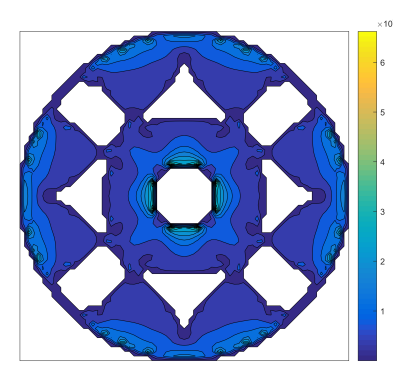

(b) Final

Fig. 7. Strain energy distributions for the baffle with design-dependent loading for the initial and BESO-found designs of the compliance minimisation test case.

The strain energy density of the initial topology is significantly higher compared to the final topology. The regions of concentrated strain energy in the initial topology are more severe, having a larger difference compared to the rest of the material. The compliance of the initial topology is $5.13 \times 10^{9} \mathrm{Nm}$, whereas, for the coupled topology, the compliance is reduced to $2.281 \times 10^{9} \mathrm{Nm}$. Thus, the optimiser has produced a final topology that uses the material of the baffle much more efficiently. Hence, the optimiser with the fluid-structure coupling is 
able to improve the design of the baffle, reducing the compliance and energy concentrations in the structure.

It is important to note that, for the final topology with fluid-structure coupling present, the structural symmetry, and hence fluid loading about the $\mathrm{x}$ and y-axes, becomes identical (Fig. 7). The initial topology is symmetric about both the $x$ - and y-axes (Fig. 7a), but not in the same way, i.e. if the topology is rotated $90^{\circ}$ it does not remain the same. Hence, initially the loading is not symmetric about the $\mathrm{x}$-axis in the same way as it is about the y-axis. Therefore, if the coupling was not present, the loading would not be updated, and this difference in the symmetry would remain present for the entire analysis. Thus, the final topology would remain symmetrical about both the $\mathrm{x}$ - and $\mathrm{y}$-axes. This is known as 2-fold rotational symmetry, $\mathrm{SO}(2)$, about the centre point. However, the coupled solution updates the fluid mechanics, and hence the pressure loading on the structure. Since there is no physical difference about the horizontal and vertical axes, there is nothing present to introduce asymmetry into the topology. Therefore, it makes physical sense for the fully coupled solution to have 4-fold rotational symmetry, $\mathrm{SO}(4)$, about the centre point. Hence, from a physical standpoint, the coupled topology is shown to be optimal.

The convergence history for the topology optimisation problem with a designdependent pressure loading is given in Fig. 8. Due to the fluid-structure coupling, steep drops and jumps in the objective are observed when the updated topology is run back through the fluid solver. It is found that initially the step changes in the objective are larger, i.e. in the earlier iterations through the optimiser, since larger changes in the topology occur in the first iterations, whereas near convergence only small changes occur. Thus, in the first few iterations the design, and hence pressure load, changes significantly resulting in large differences in the objective function (Eq. 26). The sequence takes 179 iterations to converge. Since the convergence criterion assesses the change in the objective in the previous 10 iterations (Sect. 4.3), a minimum of 10 LBM runs are performed for the convergence of the algorithm. This results in a tougher convergence as the change in the pressure loading from the previous 10 iterations 


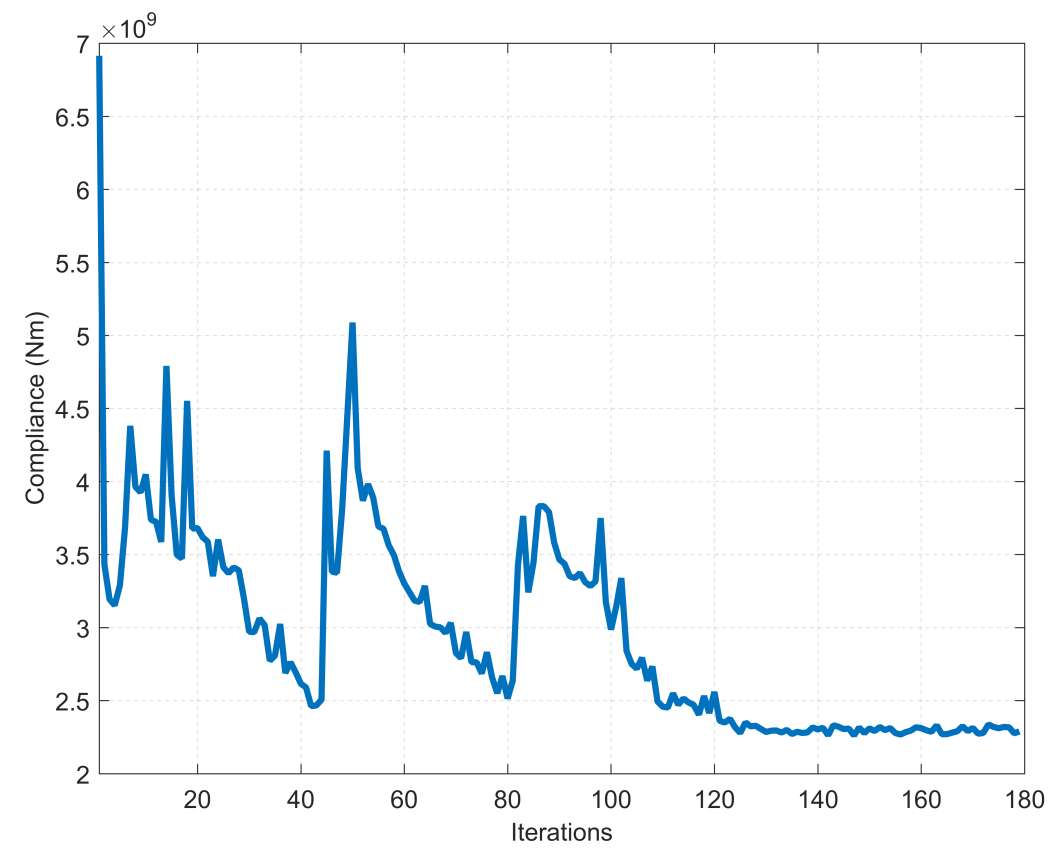

Fig. 8. Convergence history for the baffle with design-dependent loading using BESO for the compliance minimisation test case.

must also be minimal. Consequently, for the convergence of the algorithm, the last 10 LBM runs are considered. Hence, both the objective (structural) and pressure loads (fluid) must demonstrate minimal change for convergence to be achieved. Therefore, both the fluid and structural physics are converged not just the structural, as would be the case for non-design dependent loads.

It is important to note that once the fluid dynamics converge, so too do the structural dynamics of the system. This is seen in the convergence history (Fig. 8) by observing that, as the sequence progresses, the jumps in the objective 575 are reduced. This is most clearly seen in the latter iterations (Fig. 8), where, after iteration 120, the history flattens out and the update runs, through the LBM, produce very small differences in the objective. Therefore, it is plausible that a looser coupling, where the LBM is solved less often, may result in complementary final topologies, as both the fluid and structural domains converge, 
resulting in a similar final loading and hence topology. This would be a significant benefit of the proposed algorithm. If increasing the degree of coupling only results in small improvements in the objective, albeit at a large computational penalty, then lower degrees of coupling could be implemented with only small sacrifices in the quality of the objective and with large improvements in computational efficiency. This becomes significant when several designs are being considered at the conceptual design stage. For the sake of brevity, the testing of this hypothesis is left for future work, hence the focus of this study is on the development of the algorithm and physical analysis of the system.

The initial structure has a compliance of $5.13 \times 10^{9} \mathrm{Nm}$, so the compliance is reduced by approximately $56 \%$ when using the coupled BESO algorithm presented in this article. Furthermore, the main computational burden comes from the LBM runs, since one LBM run takes approximately $3000 \mathrm{CPU}(\mathrm{s})$, compared with $8 \mathrm{CPU}(\mathrm{s})$ for the optimisation routine per optimisation run. Hence, to achieve convergence the BESO algorithm takes approximately 6 days, 4 hours and 48 minutes when performed on an Intel ${ }^{\circledR}$ Core $^{\mathrm{TM}}$ i7-2720QM CPU architecture @ $2.20 \mathrm{GHz}$ when using 4 cores in parallel for the LBM simulations. Therefore, at this computational expense, the algorithm is not suitable for use at the preliminary design stage where hundreds, or even thousands, of designs are being considered. However, more computationally efficient methods could be implemented, with only a small number of designs being run through the fully coupled BESO algorithm to determine the final design.

\subsection{Vorticity maximisation}

The final analysis of this study presents the results of the topology optimisation algorithm (Sect. 5.3) for the fluid optimisation problem. First, the initial topology is presented, along with the results from a Tabu Search (TS) meta-heuristic algorithm on the same problem, used for comparison. The topology optimisation algorithm, with a novel sensitivity analysis, is then applied to the fluid problem, and the results are analysed and compared with the metaheuristic solutions. Finally, a brief comparison of the topologies produced by 
the two different criteria of this work is given.

To verify the novel formulation derived in Sect. 5.3 for the maximisation of vorticity in the flow, using a BESO algorithm, a TS algorithm was applied to the optimisation problem. The TS algorithm used a level-set parameterisation to maximise the vorticity and minimise the pressure difference of the flow. Again, the centre hole was designated as non-designable material. The Reynolds number of the flow was constrained to $R e_{0}=100$, and the volume fraction was set to have a maximum value of $V=0.8$, the same value used in the BESO algorithm. The corresponding Pareto front is displayed in Fig. 9.

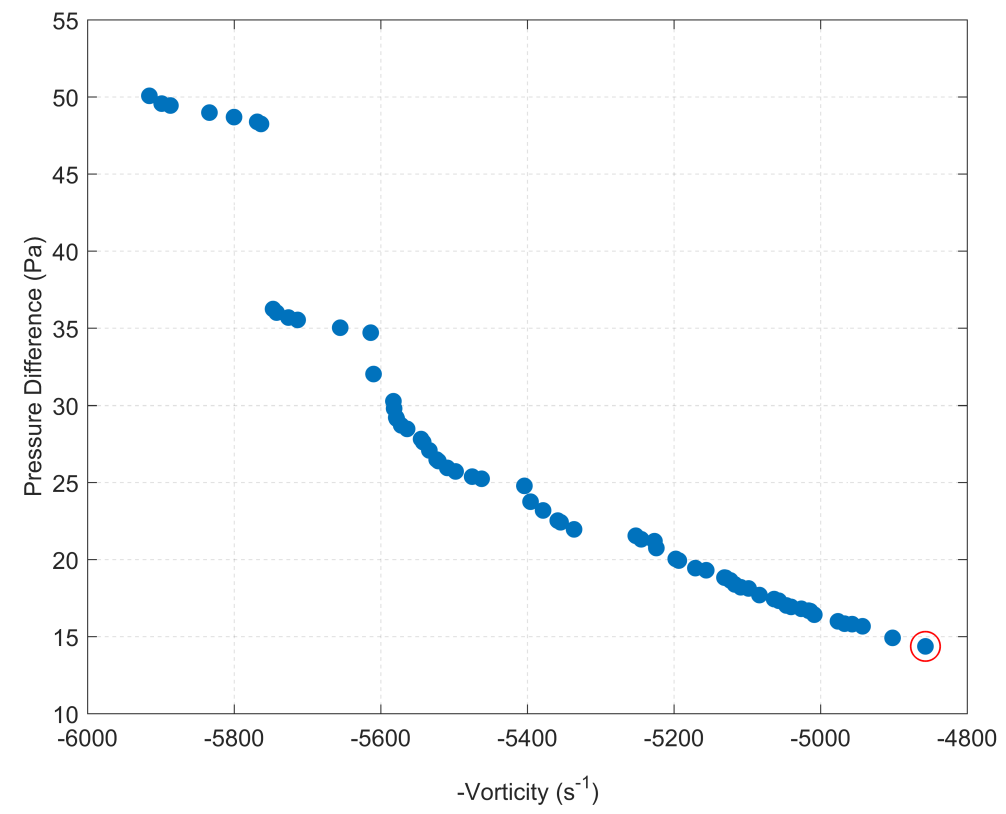

Fig. 9. Pareto front produced by the TS algorithm for the vorticity maximisation design (data produced by Dr Tiziano Ghisu).

The Pareto front (Fig. 9) shows a clear trade-off between vorticity and pressure difference. As the pressure difference is increased so is the vorticity. The maximum vorticity achieved using TS is $5915 \mathrm{~s}^{-1}$ in $500 \mathrm{LBM}$ evaluations. To ensure the topology optimisation algorithm does not have a clear advantage over the TS method, the starting topology is chosen as one of the previous solutions 
from the TS optimisation. The solution chosen is the one with the lowest vorticity, so that the starting topology is not near the final solution. The chosen solution is indicated in Fig. 9 as the data point with a circle around it. The initial topology thus has a vorticity of $4856 \mathrm{~s}^{-1}$. The volume fraction, $V=0.8$, and Reynolds number, $R e_{0}=100$, are maintained from the TS optimisation. The optimisation parameters used are: evolutionary ratio, $E R=0.02$; maximum addition ratio, $A R_{\max }=0.02$; error tolerance, $\delta=0.001$. The initial and final structures for the fluid topology optimisation problem are shown in Fig. 10.

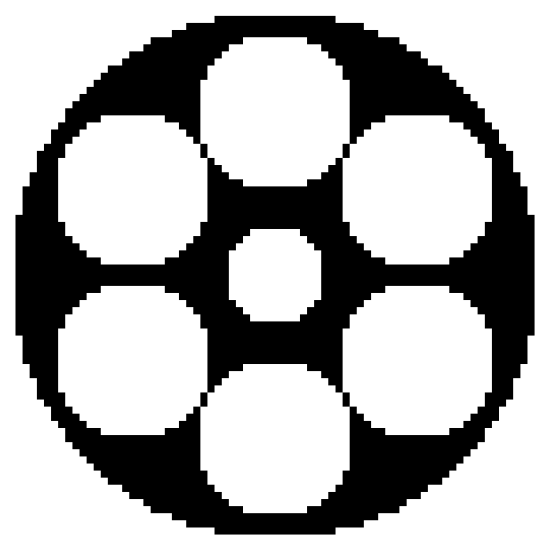

(a) Initial

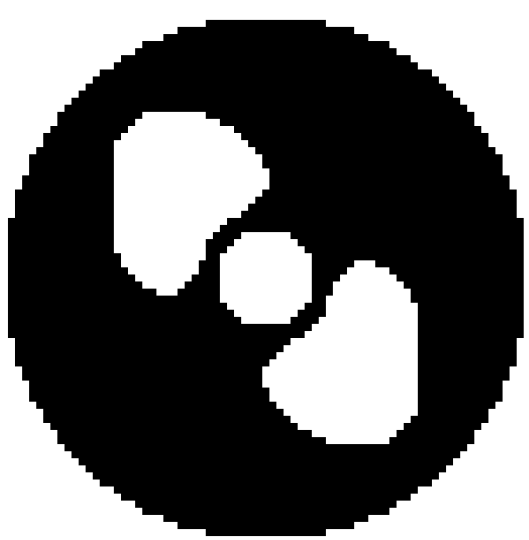

(b) Optimal

Fig. 10. Initial and final topologies for maximum vorticity.

The initial baffle design (Fig. 10a) has large holes, which allows the fluid to flow past the baffle almost unimpeded. Hence, the pressure difference is low, but so too is the vorticity. The final topology (Fig. 10b) displays a much more interesting design: the baffle becomes symmetrical about the $\pm 45^{\circ}$ diagonals, with two interestingly shaped holes positioned along one of the diagonals. Unlike the compliance objective, vorticity is not a symmetric phenomenon, and thus the final structure is not $\mathrm{SO}(4)$. In fact, [44] observed that the "strong symmetrical features" seen in the initial topology (Fig. 10) are disadvantageous for the effective mixing of the flows.

The convergence history for the fluid topology optimisation problem is given 


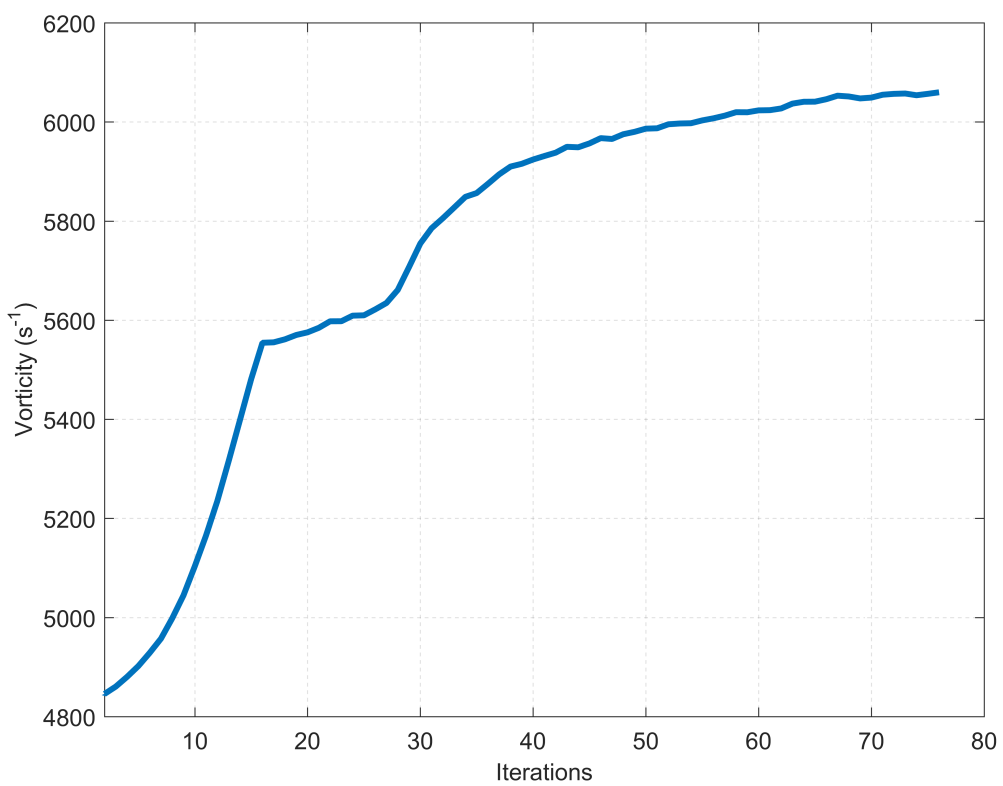

Fig. 11. Convergence history for the vorticity maximisation problem.

in Fig. 11. The first 15 iterations show a rapid increase in vorticity, as material is added to reduce the size of the fluid region. Once the volume constraint is reached, the algorithm adds and removes material, until the vorticity slowly flattens out and convergence is achieved. The solution takes 76 iterations to converge, with the final structure having a vorticity of $6060 \mathrm{~s}^{-1}$. This is a $2.5 \%$ improvement compared to the optimum found using the TS algorithm (Fig. 9 ) and a $25 \%$ improvement on the initial design. The TS algorithm, being a meta-heuristic method, is computationally restrictive compared to topology optimisation algorithms 9, requiring significantly more iterations to achieve convergence, 500 compared with 76 for the topology optimisation algorithm. Thus, such methods are confined to parametric design variables, unlike topology optimisation algorithms, which can handle 10,000 or more design variables [9]. The corresponding flow solutions for the initial design and optimal designs found by both the BESO and TS algorithms are given in Fig. 12 . 

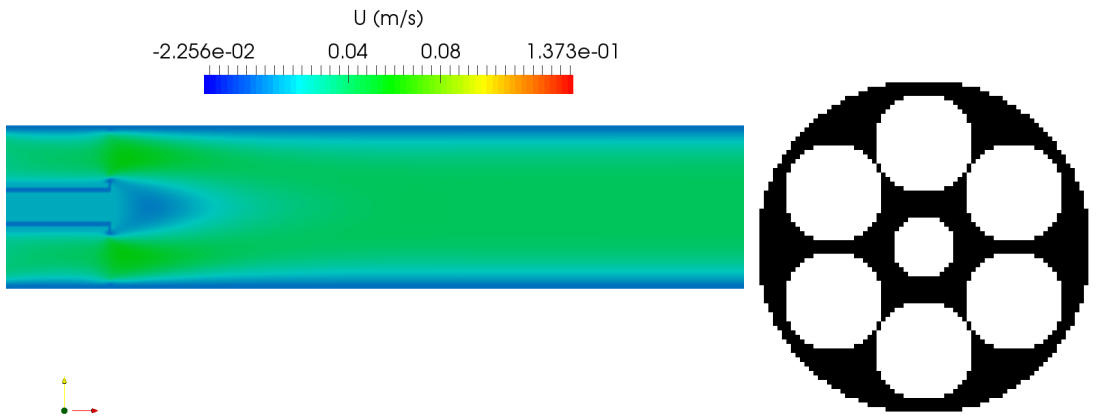

(a) Initial design

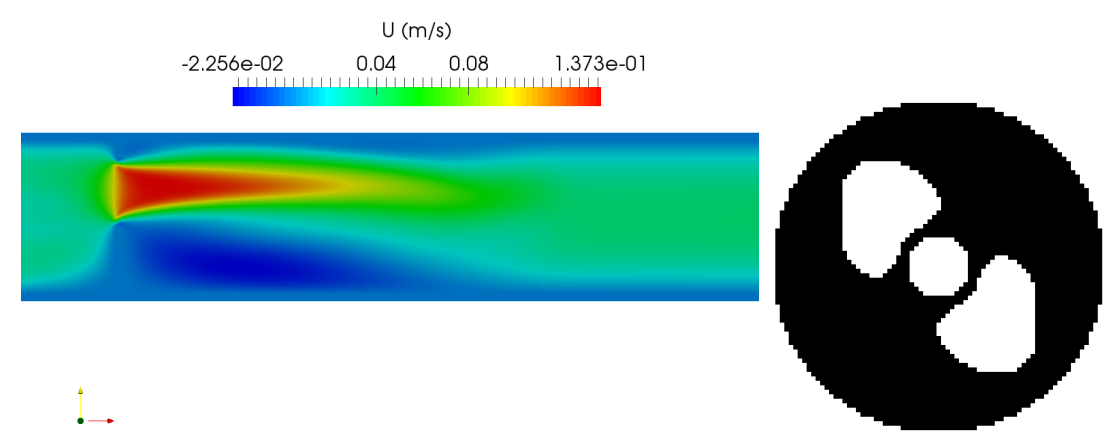

(b) Optimal design found by BESO algorithm

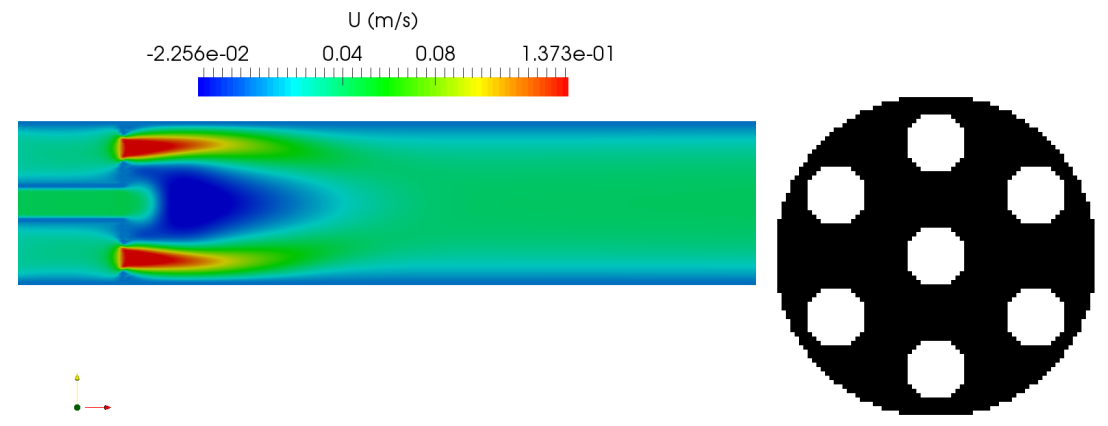

(c) Optimal design found by TS algorithm

Fig. 12. Flow patterns for the initial and optimal designs for the maximum vorticity problem.

The flow speed of the initial design (Fig. 12a is so low that it is impossible to distinguish any flow pattern(s) for the chosen scale. This is because the baffle has as little structure as possible, so as not to impede and effect the flow behaviour. Mixing is not induced by the baffle, which is why the vorticity is so 

flow patterns (Fig. 12b). As the flow approaches the baffle it is accelerated to more than double its initial velocity. This increase in energy promotes mixing downstream of the baffle where the two flows combine. This can be more clearly seen in the 3D flow visualisation plots, shown in Fig. 13. seen that the optimal baffle designs clearly promote mixing, by increasing the velocity of the flow, as suggested by 60, 44, 45. (Sect. 7.1). This is confirmed by analysing the streamlines produced by the different baffle topologies, shown in Fig. 14 . streamlines are almost unchanged before and after the baffle, having a dominant $x$ component (Fig. 14a). However, by comparing Figs. 14b and 14c it is seen that downstream of the baffle mixing is promoted. For the BESO topology (Fig. 14b), mixing occurs mainly along the $45^{\circ}$ diagonal that does not contain holes. Since the area of mixing is larger in the BESO topology, as the spacing between its two holes is larger than that between the 6 holes of the TS topology, the BESO design is able to increase the vorticity more than the TS one. Further, by comparing the flow paths for the optimised topologies using the BESO and TS algorithm (Figure 15), it is seen that the BESO design has larger recirculation zones, indicating a bigger mixing region.

Due to its flexibility and ability to explore a larger design space, the topology optimisation algorithm has produced an innovative baffle design, which surpassed the TS design. The optimiser only took 76 iterations to achieve conmany more function evaluations [66], 558\% more in this case.

Finally, it is noted that the topologies produced for the two single-objective optimisation problems of this manuscript are considerably different. This is more easily shown by comparing the strain energy distributions (SED) and flow 
objectives (Fig. 16).

Clearly, the strain energy is significantly lower for the topology with a minimum compliance objective (Fig. 16a). When plotted on the same scale (Fig. 16) the strain energy features are almost unidentifiable (Fig. 16a). In contrast, the structure with a vorticity maximisation objective has comparatively clear concentrations and portions of material where the strain energy is low. The final compliance of the two structures is $2.281 \times 10^{9}$ and $5.8494 \times 10^{9} \mathrm{Nm}$ for the compliance and vorticity objectives, respectively. It is clear that the flow is accelerated more by the baffle designed with a vorticity objective (Fig. 16d). The baffle designed with a compliance objective (Fig. 16c has less noticeable flow features when plotted on the same scale as the baffle designed with a vorticity objective. Consequently, the vorticity is $5381 \mathrm{~s}^{-1}$ and $6060 \mathrm{~s}^{-1}$ for the final designs with compliance and vorticity objectives, respectively. Thus, the objectives are conflicting. Ideally, both objectives should be considered in the design of the micro fluidic mixer, so that a trade-off can be made. A multi-objective optimisation of this sort is beyond the scope of this study and is left as future work.

\section{Conclusions}

A numerical framework for a coupled fluid-structure topology optimisation problem with design-dependent pressure loads has been presented, with the baffle plate of a micro fluidic mixing device being optimised. A BESO topology optimisation algorithm has been applied for a minimum compliance objective, with fluid-structure coupling. A BESO algorithm was developed for fluid optimisation with a maximum vorticity objective, to optimise the performance of the mixer. To the best of the authors' knowledge, this has not yet been seen in the literature of topology optimisation. The BESO algorithm was used due to its discrete nature, and hence clear description of fluid-structure boundaries. Previous studies have focused on simplified 2D problems due to the intensive computations required. The application of topology optimisation to the de- 
iterations required, with problem sizes being restricted so that acceptable computation times are achieved [12].

A coupled analysis was also performed with the BESO topology optimisation algorithm, where the pressure loads on the baffle were updated with the change the optimisation of the baffle. It was shown that the coupled algorithm was able to significantly improve the objective compared to the initial design. Furthermore, it was shown that the final design produced by the optimiser was physically acceptable, since 4-fold rotational symmetry is observed even though the initial structure has only 2 -fold rotational symmetry. Therefore, it is concluded that the fluid-structure interactions have a notable effect on the optimisation problem. This was due to the convergence of both the fluid and structural domains in the coupled analysis. Thus, it is thought that the solution may not be sensitive to the degree of coupling. It was shown that the fully coupled BESO able for evaluating many designs, as is seen at the preliminary design phase. However, it was noted that the main computational burden of the algorithm is the LBM. Therefore, it may be possible to use lower degrees of coupling, with small penalties on the objective, but at a comparatively lower computational cost. The optimisation process and solution quality can be further improved by initially setting a loose coupling and tightening the constraint by increasing the coupling once the general optimal topology has been identified. Thus, at the conceptual design phase, where several structural concepts are being considered, this analysis would become beneficial. The sensitivity of the algorithm to the degree of coupling along with other types of topology optimisation methods, i.e. SIMP and level-set, is left for future work.

Finally, a BESO algorithm with a novel sensitivity analysis for the maximisation of the vorticity in the fluid was developed. This method was compared with a TS meta-heuristic algorithm. It was found that the final structure produced 
forming the TS design by $2.5 \%$. The BESO algorithm produced an exotic and innovative final design in a relatively small number of iterations, exploring a larger design space than the TS method.

The work presented here brings high-fidelity methods, such as Lattice Boltzmann flow simulations, forward to the conceptual/preliminary design stage. Furthermore, multiple disciplines are coupled in a topology optimisation analysis to better simulate the physics of the problem to achieve the best possible designs. This type of analysis is key for the continued application of topology optimisation to real design problems, where the consideration of multiple disciplines is a very frequent requirement.

\section{Acknowledgements}

D. J. Munk thanks the Australian government for their financial support through the Endeavour Fellowship scheme.

The authors would like to thank Dr Tiziano Ghisu for producing the data displayed in Fig. 9 .

\section{References}

[1] M. Bendsøe, O. Sigmund, Topology Optimization - Theory, Methods and Applications, 2nd Edition, Berlin: Springer, 2003.

[2] D. Munk, G. Vio, G. Steven, Topology and shape optimization methods using evolutionary algorithms: A review, Struct. Multidisc. Optim. 52 (3) (2015) 613-631. doi:10.1007/s00158-015-1261-9

[3] M. Bendsøe, N. Kikuchi, Generating optimal topologies in structural design using a homogenization method, Comput. Methods Appl. Mech. Eng. 71 (1988) 197-224. doi:10.1016/0045-7825(88)90086-2.

775 [4] O. Sigmund, Design of multiphysics actuators using topology optimization - Part I: One-material structures, Comput. Methods Appl. Mech. Eng. 190 (2001) 6577-6604. doi:10.1016/S0045-7825(01)00251-1. 
[5] G. Steven, Q. Li, Y. Xie, Evolutionary topology and shape design for general physical field problems, Comput. Mech. 26 (2) (2000) 129-139. doi:10.1007/s004660000160.

[6] M. Dühring, J. Jensen, O. Sigmund, Acoustic design by topology optimization, J. Sound Vib. 317 (2008) 557-575. doi:10.1016/j.jsv.2008.03. 042 .

[7] R. Picelli, W. Vicente, R. Pavanello, Bi-directional evolutionary structural optimization for design-dependent fluid pressure loading problems, Eng. Optimiz. 47 (10) (2015) 1324-1342. doi:10.1080/0305215X.2014.963069

[8] H. Eschenauer, N. Olhoff, Topology optimization of continuum structures: A review, Appl. Mech. Rev. 54 (4) (2001) 331-390. doi:10.1115/1. 1388075 .

[9] O. Sigmund, K. Maute, Topology optimization approaches, Struct. Multidisc. Optim. 48 (2013) 1031-1055. doi:10.1007/s00158-013-0978-6.

[10] J. Deaton, R. Grandhi, A survey of structural and multidisciplinary continuum topology optimization, Struct. Multidisc. Optim. 49 (2014) 1-38. doi:10.1007/s00158-013-0956-z.

795 [11] A. Giannitsis, Microfabrication of biomedical lab-on-chip devices. a review, Estonian Journal of Engineering 17 (2011) 109-139.

[12] C. Andreasen, A. Gersborg, O. Sigmund, Topology optimization of mi口 crofluidic mixers, Int. J. Numer. Meth. Fl. 61 (2009) 498-513. doi: $10.1002 / f l d .1964$.

[13] G. Rozvany, M. Zhou, T. Birker, Generalized shape optimization without homogenization, Struct. Optimization 4 (3) (1992) 250-252. doi:10.1007/ BF01742754.

[14] V. Hammer, N. Olhoff, Topology optimization of continuum structures subjected to pressure loading, Struct. Multidisc. Optim. 19 (2) (2000) 85-92. doi:10.1007/s001580050088. 
[15] J. Du, N. Olhoff, Topological optimization of continuum structures with design-dependent surface loading - Part I: New computational approach for 2D problems, Struct. Multidisc. Optim. 27 (3) (2004) 151-165. doi: 10.1007/s00158-004-0379-y.

[20] X. Yang, Y. Xie, G. Steven, Evolutionary methods for topology optimization of continuous structures with design dependent loads, Comput. Struct.

[21] Y. Xie, G. Steven, A simple evolutionary procedure for structural optimization, Comput. Struct. 49 (5) (1993) 885-896. doi:10.1016/ 0045-7949(93) 90035-C.

[22] R. Picelli, W. Vicente, R. Pavanello, Y. Xie, Evolutionary topology optimization for natural frequency maximization problems considering acoustic-structure interaction, Finite Elem. Anal. Des. 106 (2015) 56-64. doi:10.1016/j.finel.2015.07.010. 
[23] J. Sethian, A. Wiegmann, Structural boundary design via level set and immersed interface methods, J. Comput. Phys. 163 (2) (2000) 489-528. doi:10.1006/jcph.2000.6581.

[24] S. Osher, F. Santosa, Level set methods for optimization problems involving geometry and constraints, J. Comput. Phys. 171 (1) (2001) 272-288. doi: $10.1006 / \mathrm{jcph} .2001 .6789$.

[25] G. Allaire, F. Jouve, A.-M. Toader, Structural optimization using sensitivity analysis and a level-set method, J. Comput. Phys. 194 (1) (2004) 363-393. doi:10.1016/j.jcp.2003.09.032.

[26] Z. Liu, J. Korvink, R. Huang, Structure topology optimization: Fully coupled level set method via FEMLAB, Struct. Multidisc. Optim. 29 (6) (2005) 407-417. doi:10.1007/s00158-004-0503-z

${ }_{845}$ [27] V. Challis, J. Guest, Level set topology optimization of fluids in Stokes flow, Int. J. Numer. Meth. Eng. 79 (2009) 1284-1308. doi:0.1002/nme.2616

[28] D. Munk, G. Vio, G. Steven, A bi-directional evolutionary structural optimization algorithm with an added connectivity constraint, Finite Elem. Anal. Des. 131 (2017) 25-42.

[29] S. Succi, The Lattice Boltzmann Equation for Fluid Dynamics and Beyond, 1st Edition, Oxford Science Publications, 2001.

[30] S. Wang, M. Wang, A moving superimposed finite element method for structural topology optimization, Int. J. Numer. Meth. Eng. 65 (11) (2006) 1892-1922. doi:10.1002/nme.1527.

[31] R. Cook, D. Malkus, M. Plesha, R. Witt, Concepts and Applications of Finite Element Analysis, 4th Edition, United States: John Wiley \& Sons, 2002.

[32] T. Chandrupatla, A. Belegundu, Introduction to Finite Elements in Engineering, 2nd Edition, Prentice Hall, 1997. 
[40] G. Pingen, A. Evgrafov, K. Maute, Topology optimization of flow domains using the lattice Boltzmann method, Struct. Multidisc. Optim. 36 (6) (2007) 507-524. doi:10.1007/s00158-007-0105-7.

[41] A. Evgrafov, G. Pingen, K. Maute, Topology optimization of fluid domains:

33] T. Borrvall, J. Petersson, Topology optimization of fluids in Stokes flow, Int. J. Numer. Meth. Fl. 41 (1) (2003) 77-107. doi:10.1002/fld.426

[34] L. Laniewski-Wollk, J. Rokicki, Adjoint lattice Boltzmann for topology optimization on multi-GPU architecture, Comput. Math. Appl. 71 (3) (2016) 833-848. doi:10.1016/j.camwa.2015.12.043.

[35] C. Pan, L.-S. Luo, C. Miller, An evaluation of lattice Boltzmann schemes for porous medium flow simulations, Comput. Fluids 35 (8-9) (2006) 898909. doi:10.1016/j.compfluid.2005.03.008.

[36] T. Lee, C.-L. Lin, A stable discretization of the lattice Boltzmann equation for simulation of incompressible two-phase flows at high density ratio, J. Comput. Phys. 206 (1) (2005) 16-47. doi:10.1016/j.jcp.2004.12.001

[37] Q. Liu, Q. Kang, C. Leonardi, S. Schmieschek, A. Narvaez, B. Jones, J. Williams, A. Valocchi, J. Harting, Multiphase lattice Boltzmann simulations for porous media applications, Computat. Geosci. 20 (4) (2016) 777-805. doi:10.1007/s10596-015-9542-3.

[38] Q. Li, K. H. Luo, Q. J. Kang, Y. L. He, Q. Chen, Q. Liu, Lattice Boltzmann methods for multiphase flow and phase-change heat transfer, Prog. Energ. Combust. 52 (2016) 62-105. doi:10.1016/j.pecs.2015.10.001.

[39] S. Kreissl, G. Pingen, A. Evgrafov, K. Maute, Topology optimization of flexible micro-fluidic devices, Struct. Multidisc. Optim. 42 (4) (2010) 495516. doi:10.1007/s00158-010-0526-6.

Kinetic theory approach, J. Appl. Math. Mech. 88 (2) (2008) 129-141. doi:10.1002/zamm.200700122. 
[42] G. Pingen, A. Evgrafov, K. Maute, Adjoint parameter sensitivity analysis for the hydrodynamic lattice Boltzmann method with applications to design optimization, Comput. Fluids 38 (4) (2009) 910-923. doi:10.1016/j. compfluid.2008.10.002.

[43] D. Makhija, G. Pingen, R. Yang, K. Maute, Topology optimization of multicomponent flows using a multi-relaxation time lattice Boltzmann method, Comput. Fluids 67 (2012) 104-114. doi:10.1016/j.compfluid.2012.06. 018.

[44] L. Djenidi, B. Moghtaderi, Numerical investigation of laminar mixing in a coaxial microreactor, J. Fluid Mech. 568 (2006) 223-243. doi:10.1017/ S002211200600203.

[45] B. Moghtaderi, I. Shames, L. Djenidi, Microfluidic characteristics of a multi-holed baffle plate micro-reactor, Int. J. Heat Fluid Fl. 27 (6) (2006) 1069-1077. doi:10.1016/j.ijheatfluidflow.2006.01.008.

[46] C. Cercignani, Theory and Application of the Boltzmann Equation, 1st Edition, Scottish Academic Press, 1975.

[47] P. Bhatnagar, E. Gross, M. Krook, A model for collision processes in gases. I. Small amplitude processes in charged and neutral one-component systems, Phys. Rev. 94 (3) (1954) 511-525. doi:10.1103/PhysRev.94.511.

[48] S. Chen, G. Doolen, Lattice Boltzmann method for fluid flows, Annu. Rev. Fluid Mech. 30 (1998) 329-364. doi:10.1146/annurev.fluid.30.1.329

[49] D. Wolf-Gladrow, Lattice-Gas Cellular Automata and Lattice Boltzmann Models, 1st Edition, Springer, Berlin, 2000.

[50] Y. Xie, G. Steven, Evolutionary Structural Optimization, 1st Edition, Springer, 1997.

[51] Y. Xie, G. Steven, Evolutionary structural optimization for dynami口 cal problems, Comput. Struct. 58 (6) (1996) 1067-1073. doi:10.1016/ 0045-7949(95) 00235-9. 
[52] O. Querin, G. Steven, Y. Xie, Evolutionary structural optimization (ESO) using a bi-directional algorithm, Eng. Comput. 15 (8) (1998) 1034-1048. doi:10.1108/02644409810244129.

[53] X. Huang, Y. Xie, Convergent and mesh-independent solutions for the bi-directional evolutionary structural optimization method, Finite Elem. Anal. Des. 43 (14) (2007) 1039-1049. doi:10.1016/j.finel.2007.06. 006 .

[54] X. Huang, Y. Xie, Bi-directional evolutionary topology optimization of continuum structures with one or multiple materials, Comput. Mech. 43 (2009) 393-401. doi:10.1007/s00466-008-0312-0.

${ }_{925}^{6}$ [55] D. Chu, Y. Xie, A. Hira, G. Steven, Evolutionary structural optimization for problems with stiffness constraints, Finite Elem. Anal. Des. 21 (4) (1996) 239-251. doi:10.1016/0168-874X(95)00043-S.

[56] O. Sigmund, J. Petersson, Numerical instabilities in topology optimization: A survey on procedures dealing with checkerboards, mesh-dependencies and

930 \ local minima, Struct. Optimization 16 (1) (1998) 68-75. doi:10.1007/ BF01214002.

[57] X. Huang, Y. Xie, Evolutionary Topology Optimization of Continuum Structures: Methods and Applications, 1st Edition, John Wiley \& Sons, 2010.

[58] S. Zhou, Q. Li, A variational level set method for the topology optimization of steady-state Navier-Stokes flow, J. Comput. Phys. 227 (24) (2008) 1017810195. doi:10.1016/j.jcp.2008.08.022.

[59] M. Fuchs, E. Moses, Optimal structural topologies with transmissible I loads, Struct. Multidisc. Optim. 19 (4) (2000) 263-273. doi:10.1007/ 940 s001580050123 
[60] P. Woodfield, N. Kazuyoshi, K. Suzuki, Numerical study for enhancement of laminar flow mixing using multiple confined jets in a micro-can combustor, Intl. J. Heat and Mass Transfer 46 (2003) 2655-2663.

[61] J. Romero, E. Silva, A topology optimization approach applied to laminar flow machine rotor design, Comput. Methods Appl. Mech. Eng. 279 (2014) 268-300. doi:10.1016/j.cma.2014.06.029.

[62] H. Kasumba, K. Kunisch, Vortex control in channel flows using translational invariant cost functionals, Comput. Optim. Appl. 52 (3) (2012) 691717. doi:10.1007/s10589-011-9434-y.

[63] S. Abrahamson, S. Lonnes, Uncertainty in calculating vorticity from 2D velocity fields using circulation and least-squares approaches, Exp. Fluids 20 (1) (1995) 10-20. doi:10.1007/BF00190593.

[64] C. Tsotskas, T. Kipouros, M. Savill, Fast multi-objective optimization of a micro-fluidic device by using graphics accelerators, Proc. Comput. Sci. 51 (2015) 2237-2246. doi:10.1016/j.procs.2015.05.502.

[65] D. Munk, G. Vio, T. Kipouros, G. Parks, Computational design for micro fluidic devices using a tightly coupled lattice Boltzmann and level set-based optimization algorithm, in: Proceedings of the 11th ASMO-UK Conference on Engineering Design Optimization, 2016. optimization, Struct. Multidisc. Optim. 43 (5) (2011) 589-596. doi:10. 1007/s00158-011-0638-7. 


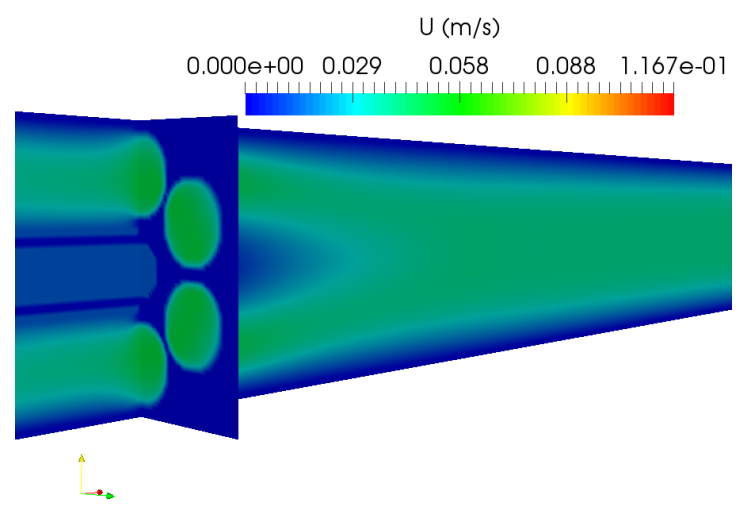

(a) Initial design

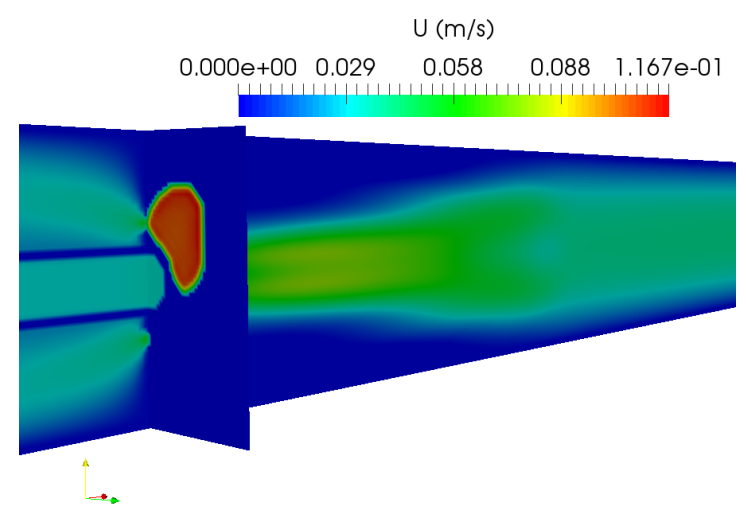

(b) Optimal design found by BESO algorithm

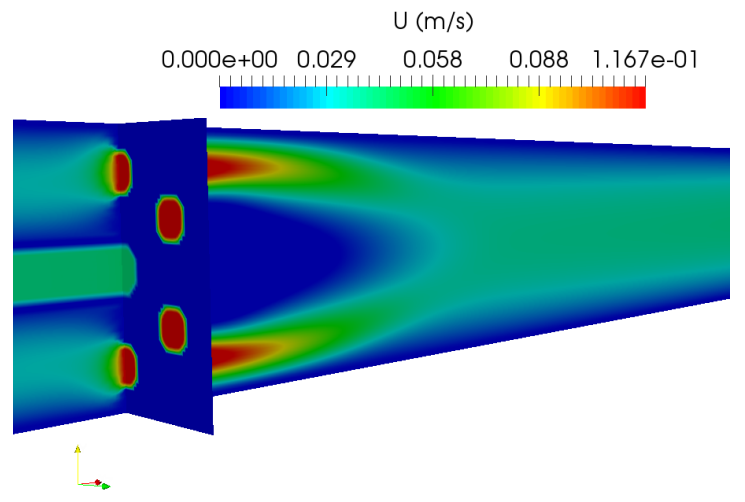

(c) Optimal design found by TS algorithm

Fig. 13. 3D flow visualisations for the initial and optimal designs for the maximum vorticity problem. 

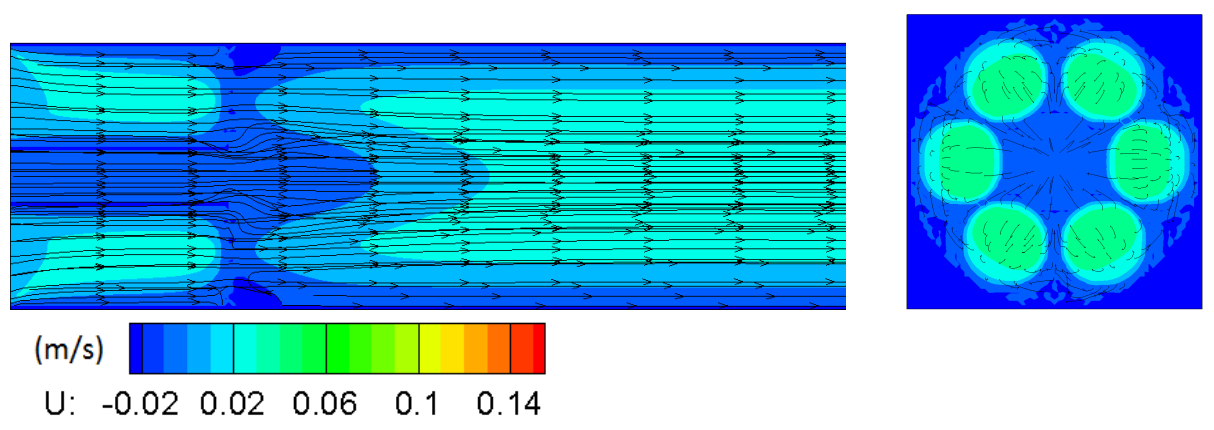

(a) Initial design
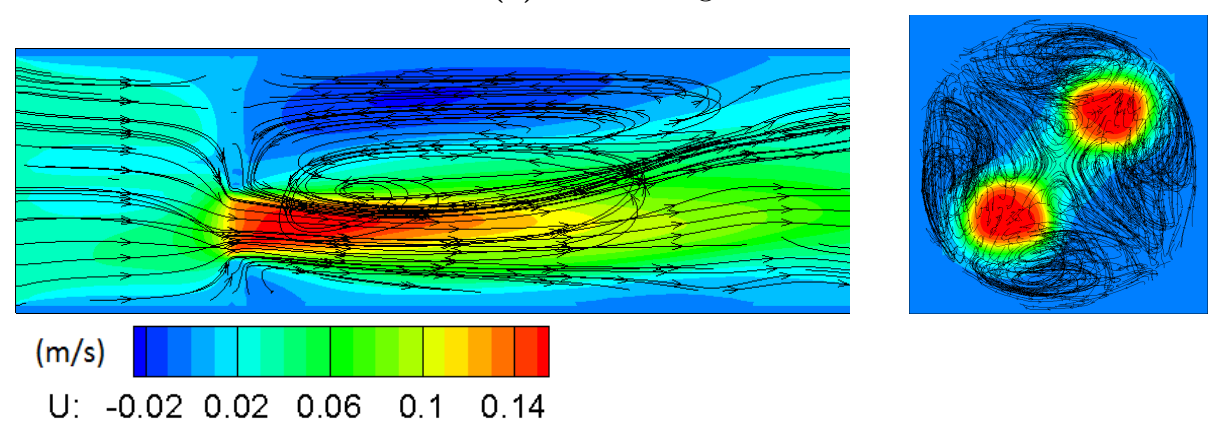

(b) Optimal design found by BESO algorithm
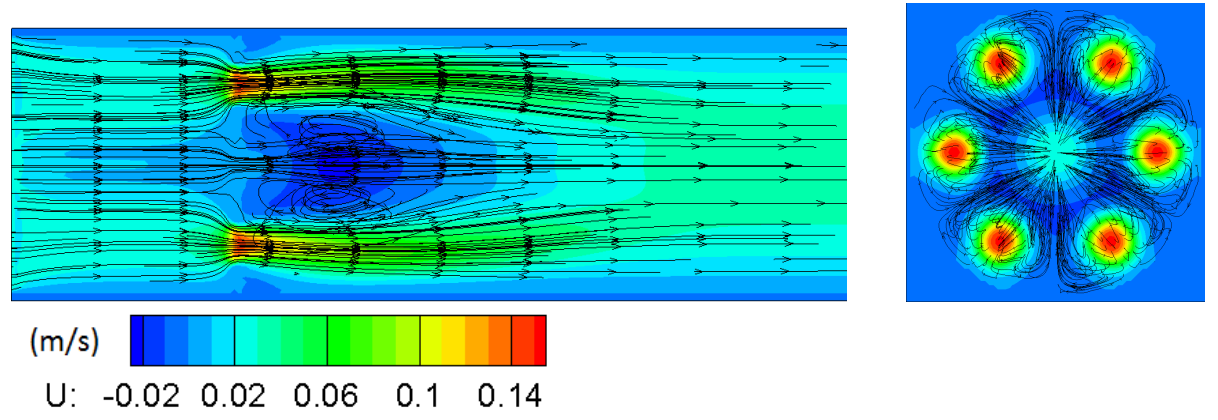

(c) Optimal design found by TS algorithm

Fig. 14. Streamlines for the initial and optimal designs for the maximum vorticity problem. 


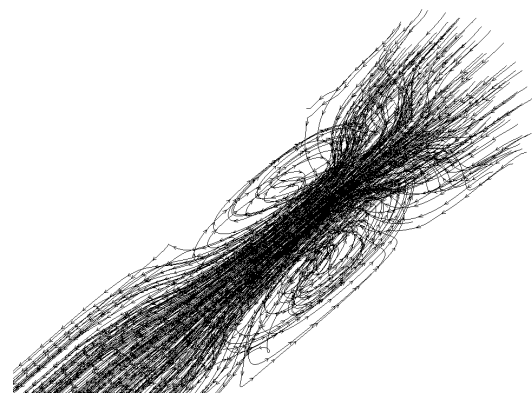

(a) Optimal design found by BESO algorithm

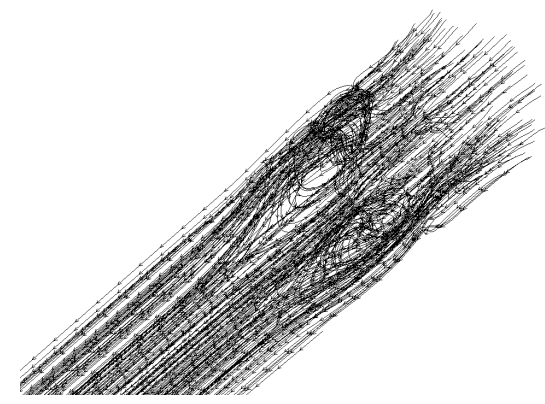

(b) Optimal design found by TS algorithm

Fig. 15. BESO and TS flow paths for maximum vorticity.

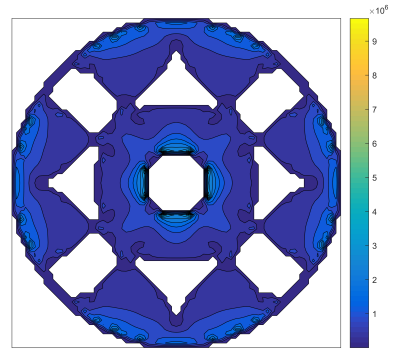

(a) SED for the compliance objective

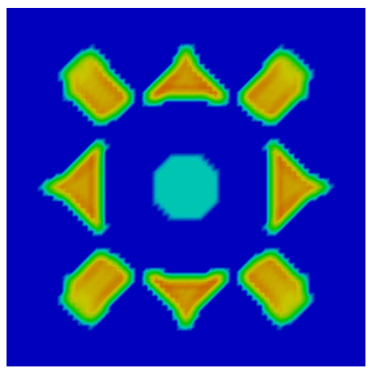

(c) Flow field for the compliance objective

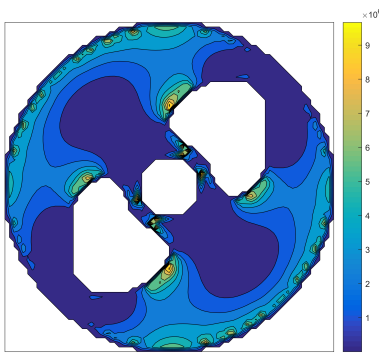

(b) SED for the vorticity objective

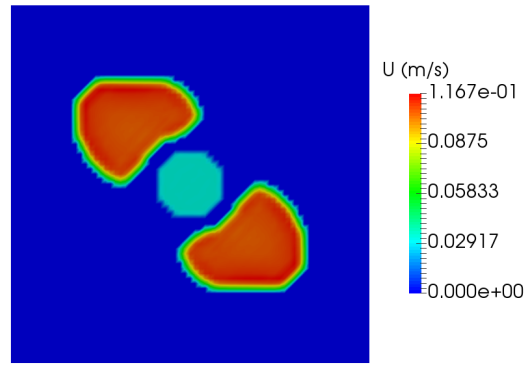

(d) Flow field for the vorticity objective

Fig. 16. Strain energy distributions and flow fields for designs found by the BESO algorithm with a compliance and a vorticity objective. 